\title{
Erratum: Statistical anisotropy in the inflationary universe [Phys. Rev. D 80, 023521 (2009)]
}

\begin{abstract}
Yuri Shtanov* and Hanna Pyatkovska
An error was made when calculating the amplitude of the perturbed scalar mode after Hubble-radius crossing. When corrected, it eliminates the leading term in the statistical anisotropy of the power spectrum, reducing it from the previously claimed upper estimate of $\lesssim 10^{-2}$ to the level of at most $10^{-6}$, which is beyond the possibility of detection. The general conclusion is that a single-field inflationary scenario cannot produce statistical anisotropy of appreciable magnitude.
\end{abstract}

\footnotetext{
*shtanov@bitp.kiev.ua
} 
An error was made when calculating the amplitude of the perturbed mode after Hubbleradius crossing, i.e., in passing from (85) to (90) and (91). An important circumstance was overlooked, namely, that the effective wave number and frequency of the mode (85) are slightly different from the corresponding quantities ( $\mathbf{k}$ and $k$ ) of the unperturbed mode, being equal to $\mathbf{k}-\delta \mathbf{k}$ and $k-\delta k$, respectively, where $\delta \mathbf{k}$ is the same as in the calculation of Appendix A and is given by (A4) for the contribution from tensor inhomogeneity. This should be taken into account when using the condition of Hubble-radius crossing for this mode. The corrected equations (90) and (91) will read

$$
A_{\mathbf{k}}(\mathbf{x})=A_{k-\delta k} \frac{(k-\delta k)^{3 / 2}}{k^{3 / 2}} e^{-\mathrm{i} \widetilde{S}_{\mathbf{k}}\left(\tau_{k}, \mathbf{x}\right)-\mathrm{i} \widetilde{T}_{\mathbf{k}}\left(\tau_{k}, \mathbf{x}\right)},
$$

and

$$
\Phi_{\mathbf{k}}\left(\tau_{f}, \mathbf{x}\right) \approx-A_{k-\delta k} \frac{(k-\delta k)^{3 / 2}}{k^{3 / 2}} e^{-\mathrm{i} \widetilde{S}_{\mathbf{k}}\left(\tau_{k}, \mathbf{x}\right)-\mathrm{i} \widetilde{T}_{\mathbf{k}}\left(\tau_{k}, \mathbf{x}\right)},
$$

respectively.

Consequently, it is necessary to make an additional correction for the amplitude $\left|A_{k}\right|^{2}$ in (A8), described by the substitution

$$
\left|A_{k}\right|^{2} \rightarrow\left|A_{k-\delta k}\right|^{2} \frac{(k-\delta k)^{3}}{k^{3}}=\left|A_{k}\right|^{2}\left[1-\frac{d \ln \left(k^{3}\left|A_{k}\right|^{2}\right)}{d \ln k} \frac{\delta k}{k}\right],
$$

which will cancel the first term in (A9). Equations (A9) and (A11) will then read

$$
\begin{gathered}
\mu_{\mathbf{k}}^{T}=Q+\left[\mathbf{n} \cdot \nabla Q-(\mathbf{n} \cdot \nabla)^{2} T_{0}\right] \frac{d \tau_{k}}{d \ln k} . \\
\nu_{\mathbf{k}}^{T}=\frac{1}{2}\left(\mu_{\mathbf{k}}^{T}+\mu_{-\mathbf{k}}^{T}\right)=Q-\frac{1}{2}(\mathbf{n} \cdot \nabla)^{2}\left[\left(T_{0}\right)_{\mathbf{k}}+\left(T_{0}\right)_{-\mathbf{k}}\right] \frac{d \tau_{k}}{d \ln k} .
\end{gathered}
$$

A similar cancellation will take place in the contribution $\nu_{\mathbf{k}}^{S}$ in (93). The leading terms written in (94) and (95) are then absent, and the numerical estimates of Secs. V-VI and in the Summary Sec. VIII are then of no relevance. The corrected expressions read

$$
\begin{gathered}
\nu_{\mathbf{k}}^{S}=-\frac{1}{2}(\mathbf{n} \cdot \nabla)^{2}\left[\left(S_{0}\right)_{\mathbf{k}}+\left(S_{0}\right)_{-\mathbf{k}}\right] \frac{d \tau_{k}}{d \ln k}, \\
\nu_{\mathbf{k}}^{T}=Q-\frac{1}{2}(\mathbf{n} \cdot \nabla)^{2}\left[\left(T_{0}\right)_{\mathbf{k}}+\left(T_{0}\right)_{-\mathbf{k}}\right] \frac{d \tau_{k}}{d \ln k} .
\end{gathered}
$$

The estimates of the terms in (95) and (96) above, made in Appendix A [see Eq. (A12) and the paragraph that follows this equation], imply that the generated anisotropy $\nu_{\mathbf{k}}=\nu_{\mathbf{k}}^{S}+\nu_{\mathbf{k}}^{T}$ of the primordial power spectrum in (122), instead of the claimed upper value $\nu_{\mathbf{k}} \lesssim 10^{-2}$, is at most of the order $10^{-6}$, which is too small to be detectable.

The general conclusion is that a single-field inflationary scenario cannot produce statistical anisotropy of appreciable magnitude. 